\title{
Use of Electroconvulsive Therapy (ECT) in Hysterical Catatonia: Case Reports of Two Women, One Young and One Elderly
}

\section{Orum $\mathrm{MH}^{* 1}$ and Aksoy $\mathrm{I}^{2}$}

${ }^{1}$ Adiyaman University Faculty of Medicine, Department of Psychiatry, Adiyaman, Turkey

${ }^{2}$ Adiyaman University Training and Research Hospital, Psychiatry, Adiyaman, Turkey

*Corresponding author: Orum MH, Adiyaman University Faculty of Medicine, Adiyaman, Turkey 02100, Tel: +90 4162161015, E-mail: mhorum@hotmail.com

Citation: Orum MH, Aksoy I (2018) Use of Electroconvulsive Therapy (ECT) in Hysterical Catatonia: Case Reports of Two Women, One Young and One Elderly. J Case Rep Stud 6(3): 306.

doi: $10.15744 / 2348-9820.6 .306$

Received Date: May 31, 2018 Accepted Date: June 25, 2018 Published Date: June 27, 2018

\begin{abstract}
Objective: To describe two cases of electroconvulsive therapy for hysterical catatonia.

Methods: A 62-years-old female patient with a diagnosis of conversion disorder and somatization disorder and a 20-years-old female patient with a diagnosis of conversion disorder were treated on electroconvulsive therapy. Electroconvulsive therapy was performed in 8 sessions with anaesthesia.

Results: 62-years-old patient began to recover after the sixth session of electroconvulsive therapy once every two days. Complete remission in catatonic symptoms also sustained during follow-up. The catatonic symptoms of the 20 -years-old patient were completely terminated after the second session of the ECT once every two days. The treatments were completed in 8 sessions.

Conclusion: We suggested that electroconvulsive therapy may be an effective treatment technique for conversion disorder earlier than drug treatment. It is thought that hysterical catatonia or another term expressing this condition may be used in psychiatric classification systems such as DSM.
\end{abstract}

Keywords: Catatonia; Conversion disorders; Electroconvulsive Therapy; Hysteria

List of abbreviations: ECT: Electroconvulsive Therapy; DSM-5: Diagnostic and Statistical Manual of Mental Disorders, $5^{\text {th }}$ Edition

\section{Introduction}

The catatonia, a clinical picture characterized by psychomotor abnormalities, was first described by Karl Ludwig Kahlbaum in 1874 [1]. In later periods, catatonia was evaluated as a subtype of schizophrenia. After the 1960s, the actual prevalence and other situations that may be related have been revealed [2]. Gelenberg (1976) stated that there are 40 cases that could cause catatonia and many new ones have been reported since then [3]. Our data suggest that catatonia is more common in mood disorders than in schizophrenia. Catatonia can also be seen in other medical conditions besides psychiatric disorders: Endocrine disorders, infections, electrolyte imbalances, epilepsy, and traumatic brain injuries can cause catatonia. Excessive doses of drugs or substances such as cocaine, ecstasy, disulfiram, and levetiracetam can also result in catatonia [4,5]. Catatonia due to conversion is a rare condition and there is insufficient information in the literature to address this issue and its treatment. In 1984, Jensen published a report dealing with conversion-related catatonia and hypnosis activity [6]. Dabholkar reported a case of hysterical catatonia in 1988 [7]. In 2012, Shah et al. wrote an article including responses of the consultants about conversion, catatonia, and culture interaction. In this report, we present two female patients suffering from hysterical catatonia and its improvement with using electroconvulsive therapy (ECT) [8].

\section{Case Descriptions}

\section{Case A}

Mrs A was 62 years old, was married for 42 years, and was in the postmenopausal period. She was being followed at a psychiatry outpatient clinic for 35 years, with a diagnosis of conversion disorder and somatization disorder. She has not been on medication for the last three months. Previously used drugs were duloxetine, venlafaxine, sulpride, olanzapine, and amisulpride. Ten days before admission, she had suddenly stopped talking and eating. There was a physical inactivity and decreased need for sleep. In history, her first complaints were weakness of muscles, pseudoseizure, and tremor. Symptoms of the disease were exacerbated 
under stress. She had previously had catatonic symptoms similar to this one and improved with an average of two months with antidepressant and antipsychotic treatment. She had no family history of psychiatric disorders. Bush-Francis catatonia rating scale score was 27 (There is no a Turkish version of this scale. We preffered the Brazilian version due to appropriate methodolgy of the article. According to this form of scale, maximum score is 69 and mean is 20.07) [9]. Physical examination of other systems were unremarkable. The urea and electrolyte, creatinine, urinalysis, liver function test, fasting blood glucose, protein level, and lipid profile were normal. Head computed tomography, brain magnetic resonance imaging, chest X-ray, and electrocardiogram were also normal. The thyroid function test was normal. There was no history of use of alcohol, cigarette and cannabis before presentation. The patient did not report any changes in her dietary and fluid intake. She was not on any drugs known to cause catatonia such as disulfiram and levetiracetam. No infection was detected. Neurological examination findings were normal. She was consulted to the neurology department, and no organic pathology was detected. She was diagnosed with conversion disorder according to Diagnostic and Statistical Manual of Mental Disorders, $5^{\text {th }}$ Edition (DSM-5) and her treatment was started with lorazepam $2.5 \mathrm{mg}$ /day and gradually increased to $10 \mathrm{mg}$ /day. Intravenous fluids were given to patient in order to reduce the risk of mortality caused by immobility, poor nutrition, and dehydration. ECT was suggested but the family did not accept it. At the end of the seventh day, the family gave ECT approval and the patient began to recover after the sixth session of ECT once every two days. Lorazepam had been stopped 24 hours (half-life is less than one day) before ECT. Complete remission in catatonic symptoms also sustained during follow-up. Written informed consent was taken from the patient in order to publish her data.

\section{Case B}

B 20-year-old female patient was admitted to Adiyaman University Training and Research Hospital because of a refusal to eat, stopped talking. There was no past psychiatric diagnosis and family history. Four months prior to admission, problems began with her brother. Common body aches, headaches, pseudoseizures appeared. Refusal to eat and physical inactivity were added to the clinical presentations. These complaints increased following the disputes. On admission, mental status examination revealed catalepsy, stupor, posturing, and mutism. Bush-Francis catatonia rating scale score was 18. Physical examination was within normal limits. The kidney, liver, thyroid functions, electrolytes, and urinalysis were all normal. Head computed tomography, brain magnetic resonance imaging, chest X-ray, and electrocardiogram were also normal. There was no history of use of alcohol, cigarette and cannabis before presentation. He was not on any drugs and no infection was detected. Neurology specialist did not have an additional suggestion. A diagnosis of conversion disorder was made according to DSM-5 and the patient was managed by lorazepam $2.5 \mathrm{mg} /$ day and gradually increased to $7.5 \mathrm{mg} /$ day in three days. The patient was managed by intravenous fluids and ECT suggestion was accepted by family. Lorazepam was stopped 24 hours before ECT started. The catatonic symptoms of the patient were completely terminated after the second session of the ECT once every two days. Written informed consent was taken from the patient in order to publish her data.

\section{Discussion}

Hysteria has a long and rich history. The first documented cases on disease known as conversion disorder extends to ancient Egypt and it belongs to Eber Papyrus in 1900 BC. The Eber Papyrus describes symptoms of seizures and a sense of suffocation. This sense of suffocation is known as globus hystericus [10]. Although it is derived from the Greek word hystera (uterus) and is conceptualized as a woman's disease caused by the "wandering womb", Freud's paper on male hysteria demonstrates this point. Hippocrates stated that when the uterus was pressurizing upward, it caused physical symptoms. In the 1600s, Sydenham left the hypocritical tradition. According to him, hysteria is not a disease but a group of diseases. Sydenham has described that stressfulevents involving hysterical melancholy can be seen as hysterical attacks, headaches, coughs, vomiting, pain in the muscles and joints. In 1794, as the English physician Ferriar noted that hysterical conversion can represent very hazardous diseases. In the 1800 s, Charcot stated like Briquet that hysteria was related to the nervous system rather than the genital system, and it might be presenting with symptoms such as paralysis, muscle contractions, anesthesias, visual alterations, mutism, pain, nausea, vomiting, and anorexia. Hysterical accidents, Janet believed, were related to ideas and suggestion as well as to a "dissociation of consciousness." The hysteria now includes symptoms related to the emotional life of the patient, such as when Janet and Freud were on time. Conversion disorder was first used by Freud but it was not officially renamed from hysterical neurosis in the DSM-II until the DSM-III in 1980 [8]. Catatonia is a syndrome, comprised of symptoms such as motor immobility, excessive motor activity, extreme negativism, and stereotyped movements [1]. Presented as a subtype of schizophrenia in DSM-IV-TR and thus having a limited area of diagnosis, catatonia was covered under a separate title in DSM-5 and other conditions it accompanies other than schizophrenia were also mentioned (Table 1). Catatonia as a conversion/hysteria symptom is an interesting phenomenon, because there is no motor activity and no response to external stimulus during full consciousness. In DSM-5, the term conversion disorder was changed as functional neurological disorder which includes criteria's such as motor symptoms, not related to general medical condition and substance, not related to neurological disorder, and clinically significant distress or impairment in social, occupational, or other important areas of functioning or warrants medical evaluation. Conversion disorder is a diagnosis of exclusion. Conversion disorder may be present with ataxia, aphonia, mutism, paralysis, tremor, hysterical seizure, dissociative anesthesia, sensory loss, psychogenic deafness, and mixed symptoms, according to DSM-5 [11]. While catatonia may be seen as a rare symptom of conversion, it is interesting that it is not included in DSM-5. It is wondered whether the term hysterical/conversion catatonia will take place in further psychiatric classification systems. 


\begin{tabular}{|l|}
\hline 1. Catatonic disorder due to a general medical condition \\
\hline 2. Specifier "with Catatonia" for \\
\hline a. Schizophrenia \\
\hline b. Schizoaffective disorder \\
\hline c. Schizophreniform disorder \\
\hline d. Brief psychotic disorder \\
\hline 3. Specifier "with Catatonia" for current or most recent major depressive episode or manic episode in \\
\hline a. Major depressive disorder \\
\hline b. Bipolar I disorder \\
\hline c. Bipolar II disorder \\
\hline 4. Catatonic disorder not otherwise specified \\
$\quad \begin{array}{l}\text { Note: DSM-5; Diagnostic and Statistical Manual of Mental Disorders, } 5^{\text {th }} \text { Edition } \\
\text { Table 1: The Catatonia Diagnosis in DSM-5 }\end{array}$
\end{tabular}

Conversion disorder does not have a standard treatment protocol and requires a multidisciplinary approach. Psychotropic drugs, both psychodynamic and cognitive behaviour therapy are helpful in overcoming conversion disorder. Although often controversial, hypnosis is also successful in treating conversion disorder [12]. Jensen described the successful hypnotic treatment of a patient with an acute catatonic reaction [6]. Also, the author stated that conversion mechanisms may underlie some presentations of catatonia. Our case reports are valuable in that catatonic status may occur due to conversion, indicating that this condition may be seen in both early and elderly age, high morbidity due to eating rejection, delayed response to oral treatment, and rapid and high effect of ECT use. Dabholkar reported a case of hysterical catatonia developing twice in a year, and on both occasions responding to ECT [7].

\section{Conclusion}

Catatonia may occur due to conversion, and ECT may act earlier than drug treatment. It is thought that hysterical catatonia or another term expressing this condition may be used in psychiatric classification systems such as DSM.

\section{References}

1. Taylor MA, Fink M (2003) Catatonia in psychiatric classification: a home of its own. Am J Psychiatry 160: 1233-41.

2. Pfuhlmann B, Stober G (2001) The different conceptions of catatonia: historical overview and critical discussion. Eur Arch Psychiatry Clin Neurosci 251 : I4-7.

3. Gelenberg AJ (1976) The catatonic syndrome. Lancet 307: 1339-41.

4. Weder ND, Muralee S, Penland H, Tampi RR (2008) Catatonia: a review. Ann Clin Psychiatry 20: 97-107.

5. Orum MH, Yilmaz H, Bildik T, Kara MZ, Gonul AS, et al. (2017) Differential diagnosis of a patient with psychotic disorder with high creatine kinase and subfebrile fever: A case report. AMAJ 2: 23-6.

6. Jensen PS (1984) Case report of conversion catatonia: indication for hypnosis. Am J Psychother 38: 566-70.

7. Dabholkar PD (1988) Use of ECT in hysterical catatonia. A case report and discussion. Br J Psychiatry 153: 246-7.

8. Shah JL, Meyer FL, Mufson MJ, Escobar JI, Goisman RM (2012) Catatonia, conversion, culture: an acute presentation. Harv Rev Psychiatry 20: 160-9.

9. Nunes AL, Filgueiras A, Nicolato R, Alvarenga JM, Silverira LA, et al. (2017) Development and validation of the Bush-Francis Catatonia Rating Scale - Brazilian version. Arg Neuropsiquiatr 75: 44-9.

10. Merskey H, Potter P (1989) The womb lay still in ancient Egypt. Br J Psychiatry 154: 751-3.

11. Stone J, LaFrance WC Jr, Levenson JL, Sharpe M (2010) Issues for DSM-5: Conversion disorder. Am J Psychiatry 167: 626-7.

12. Rosebush PI, Mazurek MF (2011) Treatment of conversion disorder in the $21^{\text {st }}$ century: have we moved beyond the couch? Curr Treat Options Neurol 13: 255-66.

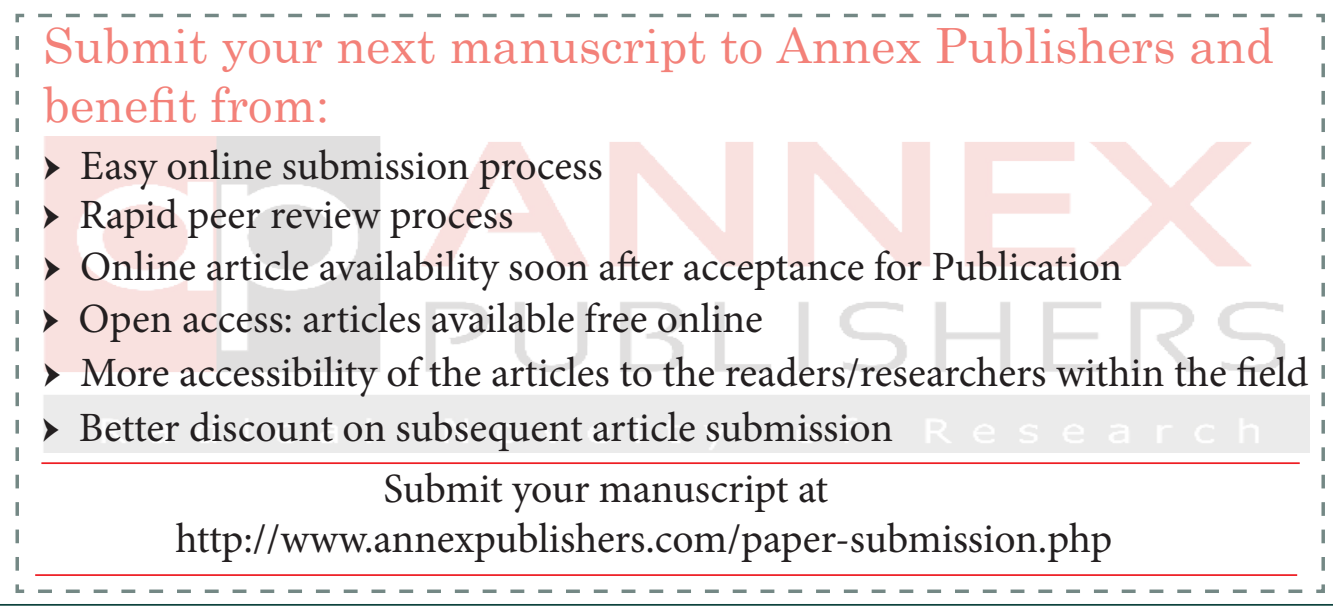

